\title{
Two-Dimensional Coulomb-Induced Frequency Modulation in Fourier Transform Ion Cyclotron Resonance: A Mechanism for Line Broadening at High Mass and for Large Ion Populations
}

\author{
Christopher L. Hendrickson, Steven C. Beu, and David A. Laude, Jr. \\ Department of Chemistry and Biochemistry, The University of Texas at Austin, Austin, Texas, USA
}

\begin{abstract}
Fourier transform ion cyclotron resonance (FTICR) spectra generated for large ion populations exhibit frequency shifts and line broadening, apparently due to Coulomb forces between ions. Although previous two-dimensional (2D) models of Coulomb effects in FTICR accounted for frequency shifts, they did not account for spectral line broadening. In this article, a 2D model is proposed that predicts line broadening due to Coulomb-induced frequency modulation. The model considers the case of two different-mass ions orbiting at their respective cyclotron frequencies around a common guiding center. A mutual modulation of the cyclotron frequency occurs at the difference frequency between ions. If the modulation period is much shorter than the FTICR observation time, then sidebands spaced at intervals approximately equal to the modulation frequency are predicted. However, if the modulation period is similar in duration to the FTICR observation period, the sidebands can no longer be resolved, which results in spectral line broadening. This latter case is a necessary consequence for isotopic peaks in the high mass region around $m / z 2000$, where deterioration in FTICR performance has been observed. Computer simulations are used to confirm the mass dependence and to demonstrate other features of the model, including a strong dependence of the modulation on ion number. In support of the model, experimental FTICR spectra for large populations of methylnaphthalene ions at $m / z 141$ and 142 exhibit constant frequency sidebands corresponding to multiples of the difference frequency for the two ions extending from nominal values of $m / z 136$ to 147. (I Am Soc Mass Spectrom 1993, 4, 909-916)
\end{abstract}

$\mathrm{F}$ Tourier transform ion cyclotron resonance (FTICR) mass spectrometry has proven to be a powerful analytical tool due to its high mass range, ultrahigh mass resolution, and multiplexing capabilities [1-3]. For example, Ijames and Wilkins [4] have reported a resolving power of 60,000 for $\mathrm{m} / \mathrm{z} 5922$ of PPG-4000 using laser desorption/ionization. However, the performance of FTICR in the analysis of biomolecules has often been disappointing. Hunt et al. [5] reported the detection of the cesium iodide cluster of $\mathrm{m} / \mathrm{z} 5848.7$ at a resolving power in excess of 30,000 but achieved less than 100 resolving power on bovine insulin at $m / z$ 5734. Efforts to obtain high mass FTICR spectra of biomolecules using matrix-assisted laser desorption have often failed to produce the expected resolution $[6,7]$, although recent results are much improved [8]. Electrospray ionization has been very successful in generating high resolution, high mass

Address reprint requests to Professor David A. Laude, Jr., Department of Chemistry and Biochemistry. The University of Texas at Austin, Austin, TX 78712.
FTICR spectra [9]. However, this has been accomplished by lowering the mass-to-charge ratio of the biomolecules to a range that is more amenable to FTICR detection.

Clearly there are complex mechanisms at work which can seriously degrade high mass performance. It is important that the forces behind these mechanisms be elucidated and corrected to maximize instrumental performance and to make high resolution FTICR spectra routinely obtainable. There is a growing body of literature detailing possible explanations for this phenomenon. Hanson et al. $\lceil 10\rceil$ have suggested that magnetic field inhomogeneities coupled with the kinetic energy of externally injected ions can cause radial diffusion of the ion cloud, resulting in loss of inn-phase coherence, thus limiting transient lifetime. Gross and co-workers [11] have described a mechanism for radial ion cloud expansion that occurs due to a parametric resonance between trapping and cyclotron oscillations. This study involved a scaling technique [12] developed by the same group which allows the characterization of high mass phenomena by studying low mass ions at a reduced magnetic field strength. 
Another explanation for resolution problems at high mass is the possibility that deleterious ion-ion interactions promote line broadening. It is commonly observed in FTICR experiments that spectra of large ion populations exhibit frequency shifts and line broadening due to Coulomb forces between ions. Modeling of Coulomb interactions has been accomplished previously by several groups [13-16]. For example, Wang and Marshall [14] have examined the interaction between ions of the same mass but different centers of gyration. Alternatively, Chen and Comisarow [15] used a point-charge model to simulate the interaction between different mass ions with a common orbit center. This model was then expanded by the authors from point-charges to charged disks [16]. These models have shown that, in two dimensions, the instantaneous position-dependent force between ions results in a mutual cyclotron frequency modulation. The average effect of this modulation is evaluated and found to produce a negative shift in the measured cyclotron frequencies of the ions. However, none of these previous two-dimensional (2D) models has accounted for spectral line broadening. Only when the models are extended in three dimensions do line broadening mechanisms emerge.

In contrast with these previous efforts to model ion-ion interactions, the present work describes the development of a 2D Coulomb line broadening model for the general case of two ions of different mass-tocharge ratio orbiting around a common center. The instantaneous position-dependent force between these ions results in a mutual cyclotron frequency modulation which occurs at a frequency equal to the difference between the ion cyclotron frequencies. When a time-dependent evaluation of this modulation is con sidered, we find that such modulation will result in spectral line broadening when the period of the frequency modulation is comparable to the experimental observation time. Such a case exists when analyzing ion populations consisting of relatively high mass ions with small differences in mass. The implications of this model for the deterioration in FTICR resolution at high mass are evaluated and comparisons are made with systems which do exhibit high resolution at high mass.

\section{Theory}

Shown in Figure 1 is a point-charge model of two different ion masses undergoing cyclotron motion with common orbit centers. The separation of these ions, $d$, is given in polar coordinates by eq 1 .

$$
d=\sqrt{\left(r_{2}-r_{1} \cos \Theta\right)^{2}+\left(-r_{1} \sin \Theta\right)^{2}}
$$

Expanding eq 1 and applying the proper trigonometric identity yields eq 2 .

$$
d=\sqrt{\left(r_{2}^{2}-2 r_{2} r_{1} \cos \Theta+r_{1}^{2}\right)}
$$

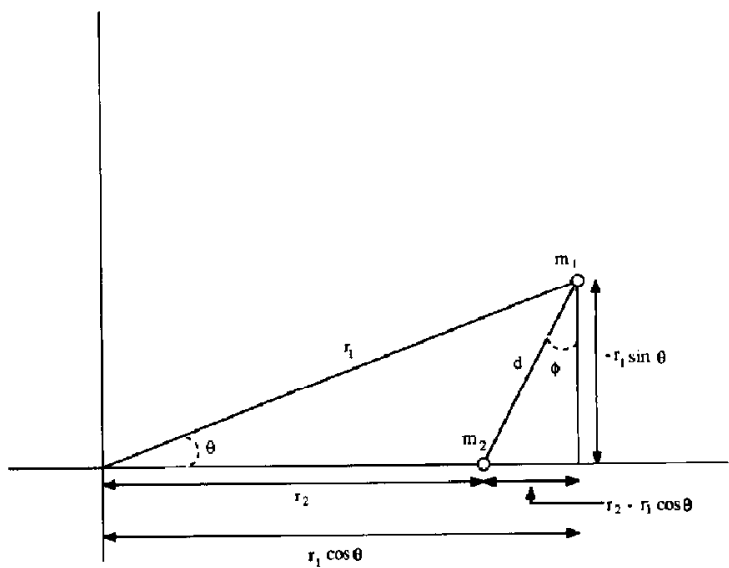

Flgure 1. Point-charge model of Coulumb elfecls. Two different-mass ions orbit around a common guiding center at their respective cyclotron frequencies with arbitrary phase and radii.

This equation gives the instantaneous distance between the ions as a function of their cyclotron radii and phase angle $(\Theta)$. This is a continuously changing distance which can be easily visualized in a coordinate frame that rotates at the cyclotron frequency of $m_{2}$ [17]. In this frame, $m_{2}$ is stationary while $m_{1}$ rotates at a frequency equal to the difference frequency between $m_{1}$ and $m_{2}$. The Coulomb force between the ions is then given by eq 3

$$
F_{\text {Coul }}=\frac{k q_{1} q_{2}}{d^{2}}=\frac{k q_{1} q_{2}}{\left(r_{2}^{2}-2 r_{2} r_{1} \cos \Theta+r_{1}^{2}\right)}
$$

where $k$ is the Coulomb constant and $q_{1}$ and $q_{2}$ are the respective charges on $m_{1}$ and $m_{2}$. This force represents the total force between the ions and can be resolved into two components, one parallel and one perpendicular to the instantaneous velocities of the ions. The parallel component of the force is orthogonal to the Lorentz force and therefore cannot perturb the iun's cyclotron frequency. However, the perpendicular component of the Coulomb force directly opposes the Lorentz force, which causes a negative shift in the ion's cyciotron frequency. The perpendicular force on $m_{2}$ due to $m_{1}$ is given by eq 4

$$
F_{\perp}=F_{\mathrm{Coul}} \sin \phi
$$

where $\phi$ is the angle between ions as defined in Figure 1. Substituting eq 3 into eq 4 gives

$$
F_{\perp}=\frac{k q_{1} q_{2}\left(r_{2}-r_{1} \cos \Theta\right)}{\left(r_{2}^{2}-2 r_{2} r_{1} \cos \Theta+r_{1}^{2}\right)^{3 / 2}}
$$

Equation 5 yields the instantaneous frequency-shifting force on $m_{2}$ due to $m_{1}$ as a function of their cyclotron radii, their charges, and the angle between ions. The 
equation is flawed, however, by the fact that the force goes to infinity for $r_{1}=r_{2}$ and $\Theta=0^{\circ}$. This condition is not possible, and instead as the magnitude of the Coulomb force approaches the Lorentz force magnitude, the ions will move apart to avoid each other so that superposition will not occur.

A more realistic model is to assume slightly different radii for $m_{1}$ and $m_{2}$. The difference between radii then becomes an important consideration. In reality it is not two point-charges that pass by one another but rather two ion clouds that pass through each other. The most satisfying physical model of this situation to date is the charged disk model of Chen and Comisarow [16]. It was shown in that work that as two disks overlap, the net radial force produced by overlapped portions of the disks is zero. The total force between disks can then be described by an "apparent" distance between unoverlapped portions of the disks. This distance is constant as the disks move through each other and is equal to the disk diameter (assuming two disks of equal size). A good approximation for this distance is the diameter of a typical ion cloud, which is on the order of $1 \mathrm{~mm}$ for the ions considered in this work. Thus we will model with $r_{1}$ and $r_{2}$ differing by that distance. However, now the direction of the radial Coulomb force depends on whether $r_{1}$ is larger or smaller than $r_{2}$. In reality there will be many ions, some with $r_{1}>\mathrm{r}_{2}$ and some with $r_{1}<r_{2}$. A simple attempt to model this situation is shown in Figure 2. Here, $m_{2}$ orbits around the center track with charge $q_{2}$ while the perturbing ion $\left(m_{1}\right)$ is split into two equal pieces, each with charge $1 / 2 q_{1}$, one with $r_{1}>r_{2}$ and one with $r_{1}<r_{2}$. Equation 5 then becomes

$$
\begin{aligned}
F_{\perp}= & \frac{k q_{1} q_{2}}{2} \\
& \times\left(\frac{\left(r_{2}-\left(r_{1}+\Delta r\right) \cos \Theta\right)}{\left(r_{2}^{2}-2\left(r_{1}+\Delta r\right) r_{2} \cos \Theta+\left(r_{1}+\Delta r\right)^{2}\right)^{3 / 2}}\right. \\
& \left.+\frac{r_{2}-\left(r_{1}-\Delta r\right) \cos \Theta}{\left(r_{2}^{2}-2\left(r_{1}-\Delta r\right) r_{2} \cos \Theta+\left(r_{1}+\Delta r\right)^{2}\right)^{3 / 2}}\right)
\end{aligned}
$$

where $\Delta r$ is the difference in cyclotron radius between $r_{1}$ and $r_{2}$. Equation 6 yields a finite frequency-shifting force over all $\Theta$ and thus provides a time-dependent analytical expression from which a shift in cyclotron frequency can be obtained. The frequency shift is negative, which can be understood by examining Figure 2 and noting that the perturbing charges always exert an outward radial force on $q_{2}$ (except for a small portion of the orbit of the outer perturbing charge). This net outward radial force on $q_{2}$ subtracts from the Lorentz force, thus causing a negative frequency shift. If it is assumed that any change in the net radial force is small with respect to the Lorentz force, then the radial

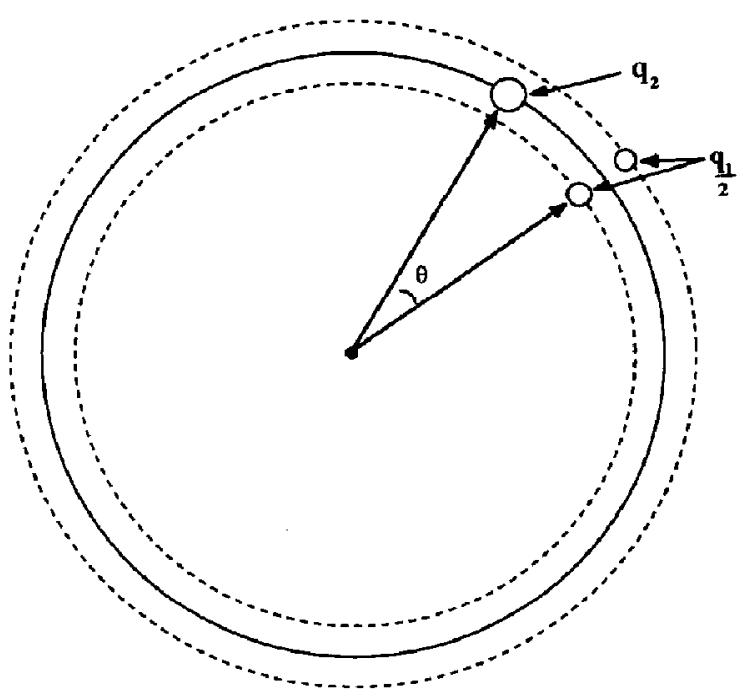

Figure 2. Split-charge model of Coulomb effects. To avoid an infinite force for $r_{1}=r_{2}$ and $\Theta=0$, the perturbing ion $\left(q_{1}\right)$ is split into two equal pieces, each with a slightly different radius than the ion of interest $\left(q_{2}\right)$.

force can be equated with the negative frequency shift of $m_{2}$ by eq 7

$$
F_{\perp}=\Delta \omega_{2} q_{2} r_{2} B
$$

where $\Delta \omega_{2}, q_{2}$, and $r_{2}$ are the frequency shift, charge, and cyclotron radius of $m_{2}$ and $B$ is the magnetic field strength. Rearranging yields eq 8 , a time-dependent analytical expression for the negative shift in the cyclotron frequency of $m_{2}$.

$$
\Delta \omega_{2}=\frac{F_{\perp}}{q_{2} r_{2} B}
$$

Equation 8 allows a time domain signal to be generated which contains this time-dependent frequency modulation. The waveform can then undergo FT to examine the frequency domain spectrum where the effects of this space-charge-induced frequency modulation will be more apparent.

\section{Experimental}

All the simulated data were generated using an $\mathrm{HD}$ 486 personal computer (High Definition Systems, Sunnyvale, CA) equipped with W.A.V.E. Version 1.11 (Vespine Software, Urbana, IL), a commercial software package that provides a waveform generation and processing environment. A program was written which utilizes this environment to model both the perturbed and unperturbed cyclotron transients of selected mass ions and displays a magnitude-mode discrete FT of these waveforms. The perturbed cyclotron frequency was calculated by subtracting the space-charge-in- 
duced frequency shift (eq 8) from the ion's true cyclotron frequency as a function of time. All simulations were done using typical FTICR parameters which included a $3.0 \mathrm{~T}$ magnetic field strength and an ion orbital radius of $1 \mathrm{~cm}$. The difference between cyclotron radii of the test charge and the perturbing charge was held constant at $1 \mathrm{~mm}$. An exponential damping constant, $\tau$, was applied to the transients and the observation period was set at $3 \tau$ in all cases. The program allows for easy variation of all experimental parameters including ion masses, number of data points, bandwidth, ion number, and damping constant.

The experimental FTICR spertra were generated with an FTICR spectrometer that included a $3.0 \mathrm{~T}$ superconducting magnet, a 2-inch cubic trapped ion cell, and ultrahigh vacuum chamber maintained at a background pressure in the low $10^{-9}$ torr range. Millipore Extrel Instruments (Madison, WI) FTMS software version 6.0 installed on a Nicolet Analytical NMC 1280 computer was used for data acquisition and processing.

FTICR spectra were acquired for methylnaphthalene which was leaked into the analyzer chamber to a pressure of $9 \times 10^{-8}$ torr. A $13 \mathrm{eV}$ electron beam operated at various emission currents and beam event times was used for ionization. Electron ionization (EI) products other than ions at $m / z 141$ and 142 were ejected from the cell prior to detection. A $2.0 \mathrm{~V}$ trap plate potential was used. The $m / z 141$ and 142 ions were excited for detection with a 100 us, $60 \mathrm{Vpp}$ radiofrequency pulse applied at a frequency midway between the $m / z 141$ and 142 ions. Broadband detection was performed with sufficient resolution to distinguish the sidebands.

\section{Results and Discussion}

The ability to model time-dependent frequency modulation of the time domain ICR signal provides a unique opportunity to examine the effect of this modulation on the observed ICR frequency and linewidth. Figure 3 shows the radial Coulomb force on $m_{2}$ as a function of the position angle $\Theta$ (which is a function of time). This was obtained by plotting eq 6 as a function of $\Theta$. The shape of the modulation is very similar to results presented in previous work by Chen and Comisarow [16]. This force modulation results in a corresponding ICR frequency modulation. Since some type of frequency modulation must certainly occur in the FTICR experiment, it is important to consider the likely consequences of such modulation. The general effects of a simple sinusoidal frequency modulation are shown in Figure 4. When no modulation occurs, as in Figure 4a, FT yields a lorentzian lineshape with a linewidth characteristic of the observation period. Figure $4 b$ depicts a modulation that completes many cycles during the observation period. The FT of this type of transient yields a spectrum similar to that of Figure 4a, with the

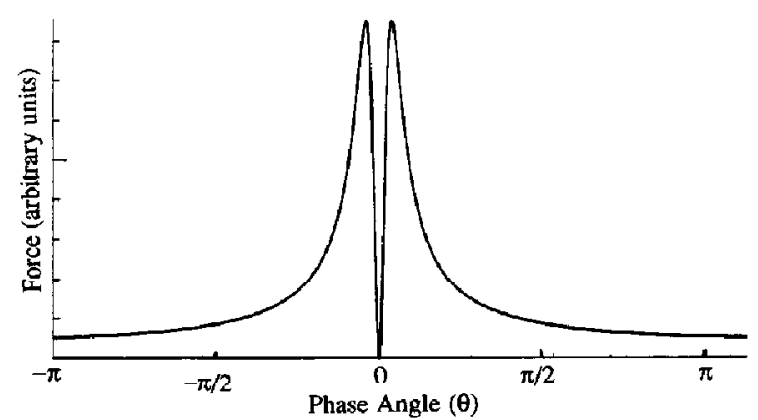

Figure 3. Radial Coulomb force on $m_{2}$ due to $m_{1}$ as a function of the position angle $\Theta$ (see Figure 1). The force also depends on ion number and radius. This force produces an identically shaped frequency modulation.

addition of sidebands, spaced at intervals equal to the modulation frequency, which remove amplitude from the central peak. However, if only one cycle of the modulation is completed during the observation period, as in Figure $4 \mathrm{c}$, the sidebands can no longer be resolved. This effectively broadens the peak of interest.

The appearance of sidebands in the frequency spectrum is a consequence of any type of frequency modulation [18]. For a simple sinusoidal modulation such as the one shown in Figure 4, the amplitude of the sidebands is a function of the modulation index, which is described by eq 9

$$
M=\Delta \omega / f
$$

where $\Delta \omega$ is the maximum frequency shift from the true cyclotron frequency of the ion and $f$ is the frequency of the modulation between ions. The amplitudes of the sidebands vary as Bessel functions of integral order of the first kind and are spaced at
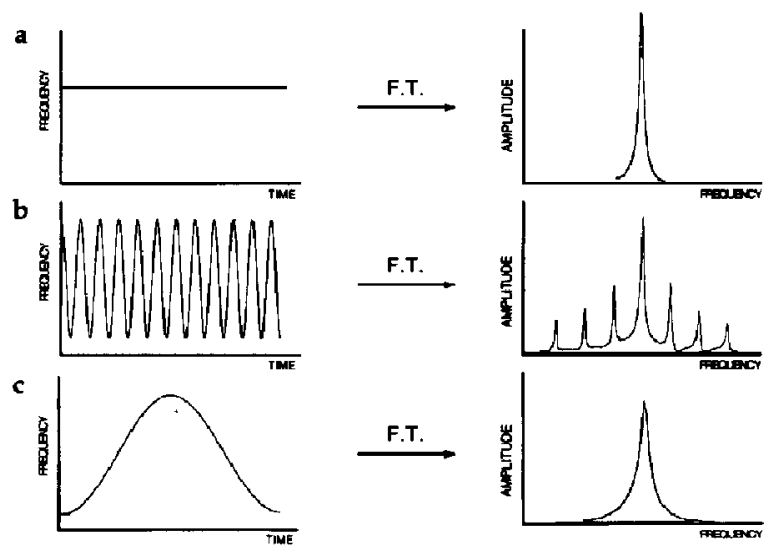

Figure 4. General effects of frequency modulation on peak shapes for (a) a constant frequency, (b) a modulation period much shorter than the observation period, and (c) a modulation period equal to the observation period. Line broadening is observed only when the modulation period is comparable to the observation period. 
intervals of $f$ from the peak of interest. For the more complicated modulation presented in Figure 3, no such simple relationship exists. The modulating wave can be broken down into its Fourier components and treated as an ensemble of sinusoidal modulating waves [19]. However, this method is only applicable when the modulation index is small and is then only approximate. For a complicated modulation the spacing and amplitudes of the sidebands are much less predictable, with possible consequences being asymmetrical peak amplitudes and uneven sideband spacing. Although no theoretical treatment was carried out in this work, a simple empirical study showed that as the modulation index increased, the number and collective amplitude of observable sidebands also increased. Obviously the modulation index will increase when ion number increases, but it will also increase as the modulation frequency, $f$, is reduced. As a consequence, this type of spectral perturbation is more pronounced for ions which are closely spaced in frequency.

\section{Model Simulations}

The broadening predicted in Figure 4 occurs only when the period of the modulation is comparable to the observation period (it is only then that the sidebands cannot be resolved). This again implies that the frequency difference between ion packets must be very small, which explains the insignificance of this mechanism for low mass measurements $(<500 \mathbf{u})$ except when ion number is very large. In contrast, as higher mass ions are considered, this requirement becomes a natural occurrence not only because the frequency difference between ions gets smaller, but because isotope peaks are increasingly prominent in the mass spectrum. As was noted earlier, it is at approximately $\mathrm{m} / \mathrm{z} 2000$ for a $3.0 \mathrm{~T}$ magnet that high resolution FTICR spectra are no longer routinely acquired. Therefore we consider as a model a peptide with a mass of $2000 u$ with equal abundance $M$ and $M+1$ ions (this corresponds to a molecule which contains about 100 carbon atoms). The modulation period for ions of $m / z$ 2000 and 2001 is $87 \mathrm{~ms}$, a time which is comparable to a medium resolution FTICR detection event.

Shown in Figure 5 are comparison spectra of modulated and unmodulated transients, all acquired for 150 ms. Ion pairs at $\mathrm{m} / \mathrm{z} 500$ and 501, 1000 and 1001, and 2000 and 2001 are presented, with each modulated spectrum modeling the interaction of 50,000 ions. $\Lambda \mathrm{t}$ lower mass the modulation frequency is relatively large and the sidebands which are generated are clearly resolved. However, as the mass-to-charge ratio is increased, the modulation frequency becomes smaller. The sidebands then become more closely spaced and therefore harder to resolve. When the masses considered are increased to $m / z 2000$ and 2001, the period of the modulation $(87 \mathrm{~ms})$ is comparable to the observation time (150 ms) and the sidebands can no longer be

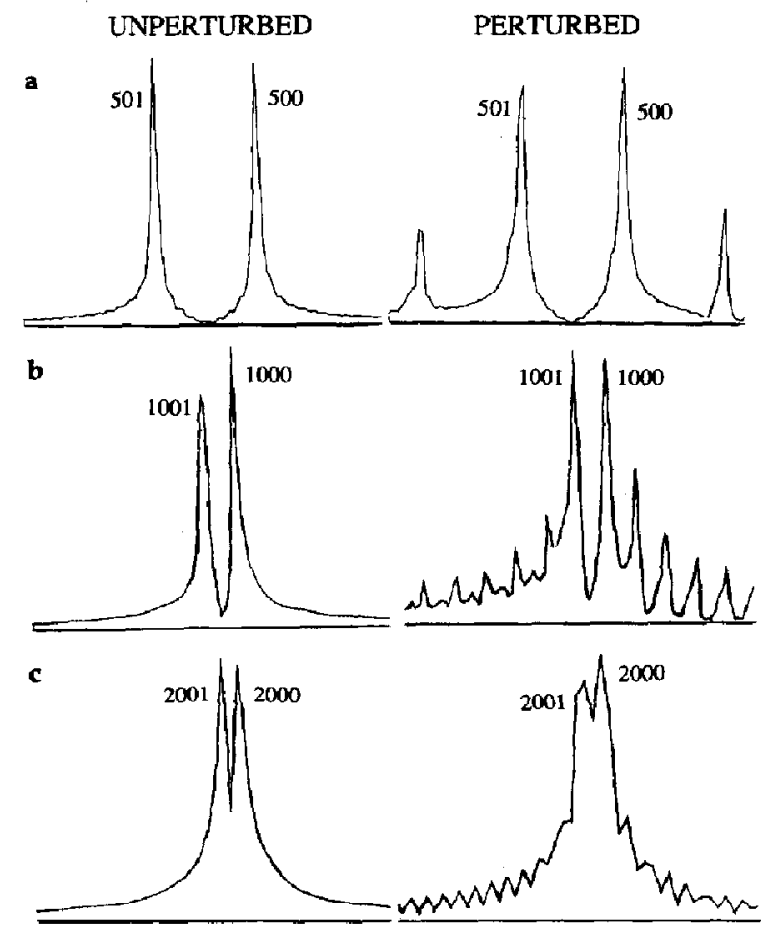

Figure 5. Computer simulated spectra of perturbed and unperfurbed ion pairs at (a) $m / z 500$ and 501 , (b) $m / z 1000$ and 1001 , and (c) $m / z 2000$ and 2001. All ion packets contain 50,000 ions and the observation period is $150 \mathrm{~ms}$ in all cases. Each of the two lower mass ion pairs produce sidebands when perturbed while the high mass ion pair, which has a modulation period of $87 \mathrm{~ms}$, exhibits substantial line broadening, in agreement with the model.

separated from the peaks of interest. This results in a broadened linewidth.

A more detailed examination of the sidebands is presented in Figure 6 , which shows the effect of increasing the acquisition period on the observed lineshapes of 50,000 interacting ions. In Figure $6 a$ the acquisition time exactly matches the modulation period. Although the two peaks are not clearly resolved even for the unmodulated case, the lineshapes are noticeably broadened due to the unresolved sidebands that are produced. If the acquisition time matches two modulation periods, as in Figure $6 \mathrm{~b}$, most but not all of the sidebands are resolved from the still broadened lineshapes. In Figure $6 \mathrm{c}$ the acquisition time has been lengthened to four modulation periods. In this case no line broadening is evident and presumably all of the sidebands have been resolved. It should be noted here that this line broadening mechanism can occur over a large range of acquisition times which need not be exact multiples of the modulation period. In fact, the frequency modulation is a continuum effect, with the sideband spacing varying from infinitely small to infinitely large. The sidebands are always present but are harder to resolve as their spacing decreases. 


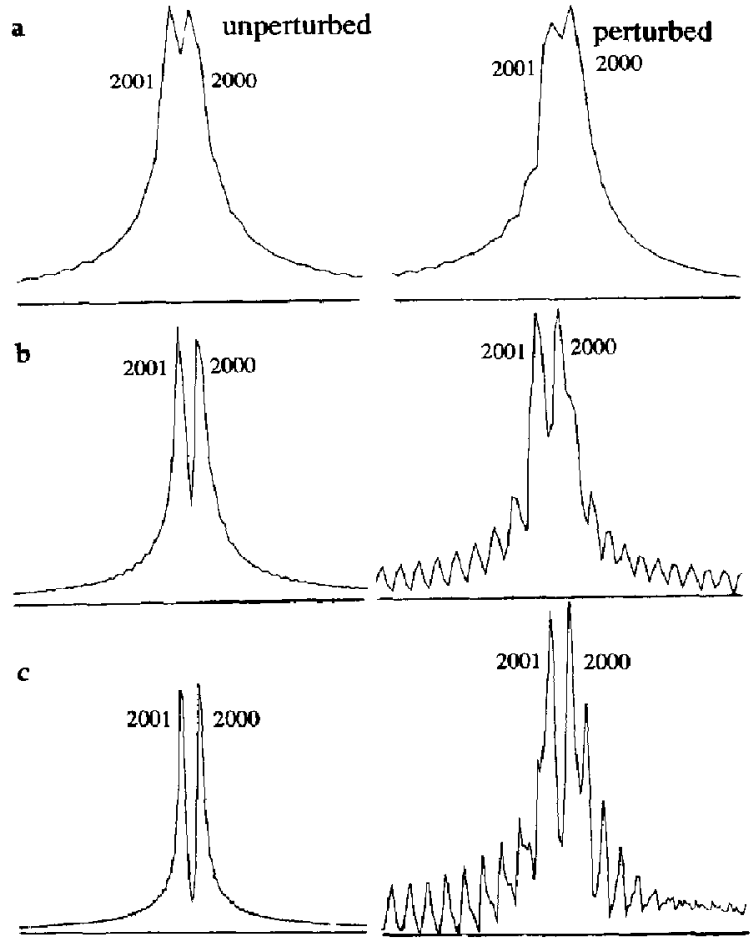

Figure 6. The effect of observation period on the observed lineshapes of the ion pair at $m / z 2000$ and 2001. Observation times are (a) $87 \mathrm{~ms}$ (equal to the modulation period), (b) $174 \mathrm{~ms}$ (twice the modulation period), and (c) $348 \mathrm{~ms}$ (four times the mudulation period). As the observation period becomes shorter, line broadening occurs because the sidebands can no longer be resolved.

Equation 6 predicts an increasing Coulomb force as the number of ions involved increases. It might then be expected that Coulomb-induced line broadening would also increase with increasing ion number. This is reflected by the model in Figure 7, where ion numbers of $0,50,000,100,000$, and 400,000 are presented. If Coulomb effects are not included, ions of $\mathrm{m} / \mathrm{z} 2000$ and 2001 are nicely resolved using a $150 \mathrm{~ms}$ acquisition time. When the Coulomb modulation is included, broadening occurs and worsens as ion number is increased. If 400,000 ions are included in each pointcharge, coalescence of the two peaks is complete.

It is appropriate at this point to consider situations in which line broadening as a result of this mechanism might not accur. This model presents the case in which only two different mass-to-charge states are considered. As more species are included, the modulation will become more complicated (superposition is not necessarily valid) with effects on the spectrum becoming equally complex. However, if enough different species are present, the modulation should decrease as the Coulomb force on a given ion packet becomes more constant with time. This may be the case for cluster formation and polymer ionization in which
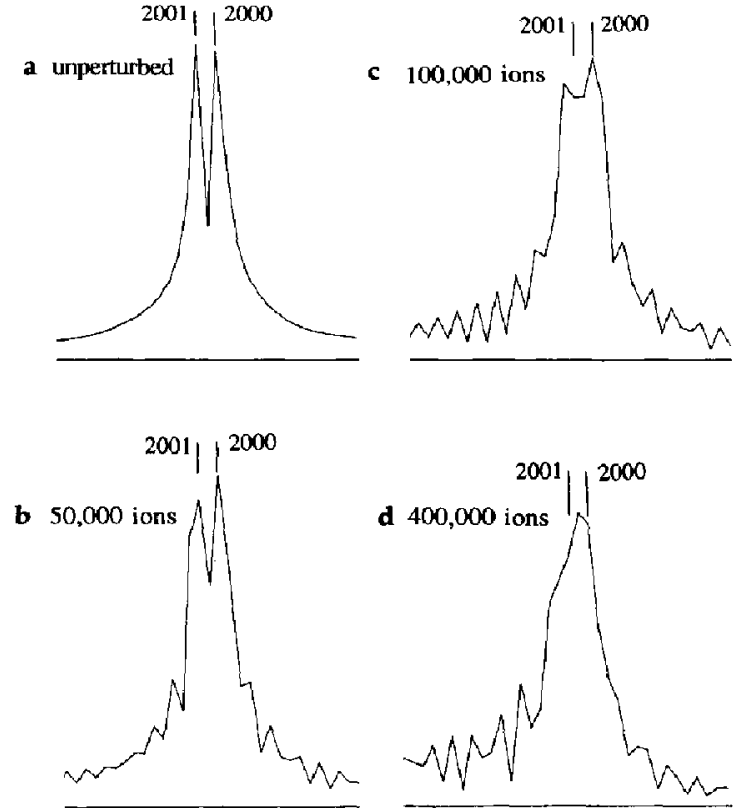

Figure 7. The effect of ion number on the observed lineshapes of the ion pair at $m / z 2000$ and 2001 . Ion numbers are (a) 0 ions (the unperturbed case), which results in two nicely resolved peaks, (b) 50,000 ions, (c) 100,000 ions, and (d) 400,000 ions, which results in total coalescence of the two peaks.

large envelopes of systematically varying mass-tocharge states are generated. In these cases so many different mass-to-charge states are generated that it is possible that no beat pattern is observed during the detection period. The lack of a beat pattern implies that modulation has not occurred. This apparent absence of frequency modulation is a consequence of motional averaging effects from the large ensemble of ion packets. The result is the near constant, timeindependent radial Coulomb force which is predicted to cause frequency shifts but no line broadening [15, 16]. Thus it is not inconsistent with the model that these ionization processes have been utilized to obtain high resolution, high mass FTICR spectra $[4,20]$.

\section{Experimental Evidence for the Model}

Conclusive validation of the model requires the observation of sidebands spaced at the difference frequency of two neighboring peaks. Experimental conditions that promote the generation of sidebands include the presence of two large ion populations closely spaced in frequency. The smaller the masses of the two ions, the larger the ion population must be to generate observable sidebands (assuming a constant mass difference). As stated earlier, the presence of other ions in the cell would tend to suppress the modulation amplitude between the two primary ions packets. 
The low energy EI spectrum of methylnaphthalene exhibits ions at $m / z 141$ and 142 with a frequency difference of $2319 \mathrm{~Hz}$. Although this difference is relatively large, we had reason to believe that a sufficient population of the two ions could be generated to observe the sidebands. Several years ago, while evaluating suspended trapping pulse sequences for alleviating space charge in the gas chromatography experiment [21], we observed that FTICR spectra of large methylnaphthalene ion populations were far more susceptible to space-charge distortion than equivalent populations of similar molecules. The only distinguishing characteristic of methylnaphthalene was the presence of two closely spaced ions in the EI spectrum. We speculated that coulombic interactions between these ion populations was responsible for the distortion which prompted the investigation that led to the model presented here.

Presented in Figure 8 are FTICR spectra of methylnaphthalene acquired under conditions which promoted a hundredfold difference in the ion population present in the cell. Presented in Figure 9 are the best matches of simulated spectra to these experimental spectra. The spectrum of the smaller ion population in Figure 8a exhibits peaks for the molecular ion, a hydride abstraction product, and a ${ }^{13} \mathrm{C}$ isotope product at $(M+1)^{+}$. A small peak corresponding to loss of two hydrogens is also observed. The spectrum of the large ion population in Figure $8 \mathrm{~b}$ exhibits the expected ions, but additionally includes peaks at nominal values ranging from $m / z 136$ to 147 . In some cases more than one peak is observed at each nominal mass-to-charge ratio value.

In interpreting the peaks from the spectrum in Figure $8 \mathrm{~b}$, there are several possible explanations. From a perspective in which the peaks correspond to detection.

\section{LOW ION NUMBER}

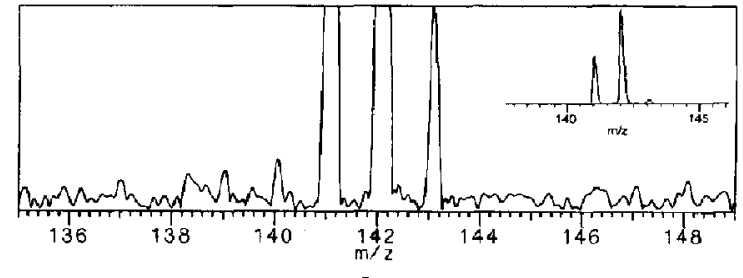

HIGH ION NUMBER

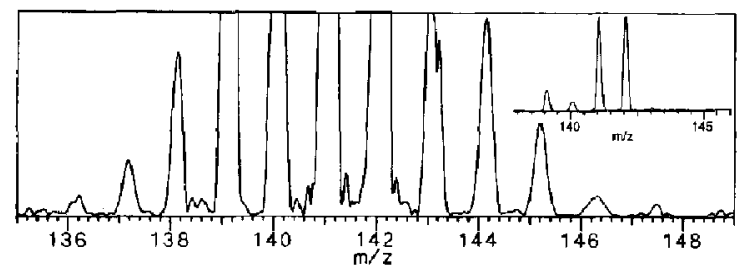

b

Figure 8. Experimental FTICR spectra of methylnaphthalene ions formed by $13 \mathrm{eV} \mathrm{EI}$ in (a) a $5 \mu \mathrm{A}, 5 \mathrm{~ms}$ beam event and (b) a $60 \mu \mathrm{A}, 50 \mathrm{~ms}$ beam event.
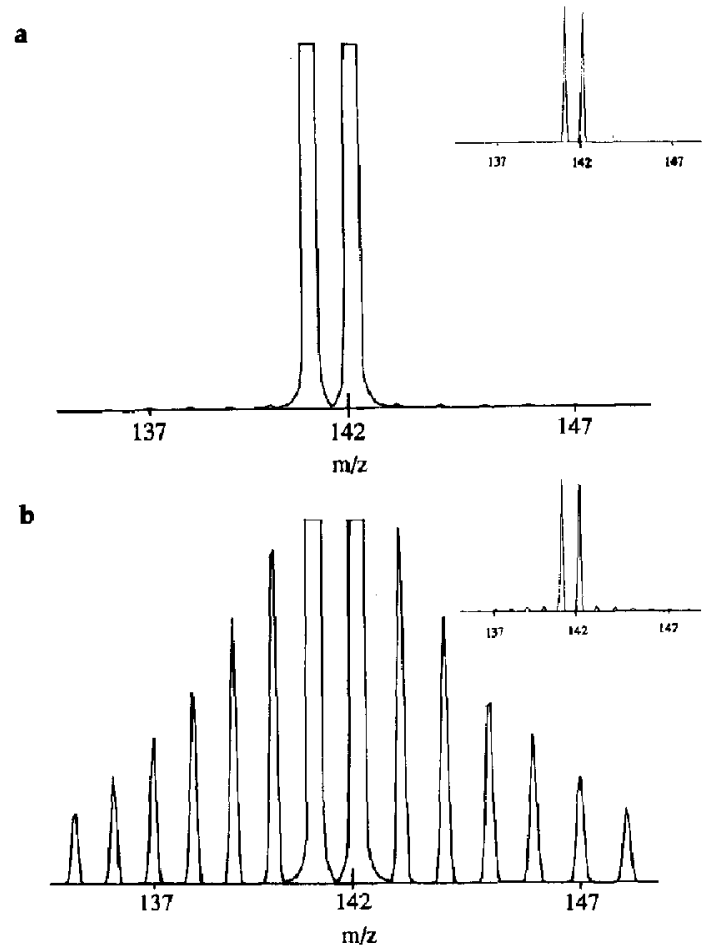

Figure 9. Simulated FTICR spectra of methylnaphthalene ions at $m / z 141$ and 142 using (a) 1000 ions and (b) 100,000 ions.

of real ions, the peaks below the molecular ion peak would correspond to loss of up to six hydrogens. The peaks above the molecular ion peak would correspond to addition of up to five hydrogens. Some contribution from ${ }^{13} \mathrm{C}$ isotope peaks would also occur. If the peaks corresponded to artifacts predicted by the Coulomb line broadening model, then the spectrum would include an envelope of sideband peaks separated by $f$ $(2319 \mathrm{~Hz}$ in this case).

The simulation program that generated the spectra in Figure 9 is not sophisticated enough to reproduce precisely the experimental data shown. The primary limitation is that the program presently accommodales only two ions when clearly ions of other masses are present in the trapped ion cell. These additional ion packets would both suppress to some extent the modulation between the two primary ions but would also contribute additional modulation peaks. Therefore it is not surprising that in some cases multiple peaks for the same nominal mass-to-charge ratio are observed.

It can be argued that all the peaks in Figure $8 \mathrm{~b}$ correspond to real ions. However, it is possible to distinguish real ionic signals from sideband peaks. Real ions corresponding to the gain or loss of hydrogens in the spectrum will be present at equal spacings corresponding to the mass of a hydrogen. Sidebands will be present at equal frequency spacings corresponding to the frequency difference of the $m / z 141$ 
and 142 peaks. The peak differences in Figure $8 \mathrm{~b}$ are plotted in Figure 10 along with predicted lines for isomass and isofrequency differences. The data clearly reject the possibility that the spectral peaks are real and instead conform to the constant frequency spacing predicted by the $2 \mathrm{D}$ line broadening model.

\section{Conclusions}

In the present work a $2 \mathrm{D}$ point-charge model is developed and demonstrated to be useful for studying Coulomb effects between ions in FTICR. By using this new model, an analytical expression is derived that describes a time-dependent Coulomb force between ions which modulates their respective ICR frequencies. This modulation is studied by computer simulation and is found to cause spectral sidebands which cannot be resolved when the period of the modulation is comparable to the observation period. Experimental evidence to validate the model is presented for a low mass, large ion population environment.

The line broadening is more likely to occur at high mass (greater than $m / z 2000$ at $3 \mathrm{~T}$ ), where the frequency difference between equally abundant isotope peaks is very small, thereby reducing the size of the population necessary to observe the effect and also allowing the period of the modulation to match a typical high resolution FTICR detection period. The model suggests that systems which produce many different mass-to-charge states, such as laser desorption ionization of polymers and cluster formation, can avoid this modulation mechanism because of motional averaging and that, consequently, high resolution, high mass spectra for these systems should be more easily generated.

Work to be done in the future will include extension of the model to three or more different-mass ions. This is important because as higher mass biomolecules

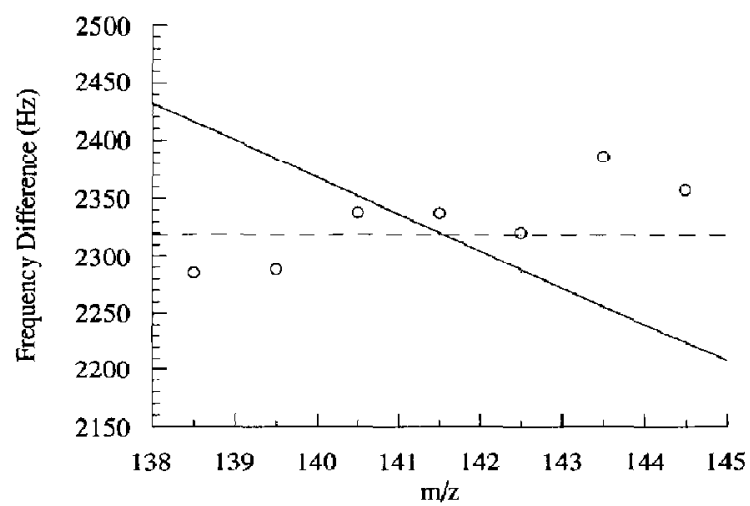

Figure 10. (o) Peak differences for adjacent nominal mass ions in Figure $8 \mathrm{~b}$ are compared to predicted curves if $\left({ }_{-}{ }^{-}\right)$the peaks represent ions separated in spacing by a single hydrogen atom or (-...) the peaks correspond to a constant frequency difference of $2319 \mathrm{~Hz}$ are considered, more isotope peaks will be prominent in the mass spectra. The complex modulations which result from multiple ion packets should allow greater insight into these interactions and their consequences on real FTICR spectra. Additional experimental verification of the mechanism for high mass ions will be attempted.

\section{Acknowledgments}

This work was supported by the Arnold and Mabel Beckman Foundation, the Texas Advanced Research and Technology Program, the National Science Foundation, and The National Institutes of Health.

\section{References}

1. Dunbar, R. C. Analytical Applications of FTICRMS; VCH: New York, 1991; chap 1.

2. Buchanan, M. V.; Comisarow, M. B. In FTMS: Evolution, Innovation and Applications; Buchanan, M. V., Ed.; ACS Symposium Series 359; American Chemical Society: Washington, DC, 1989; pp 1-20.

3. Marshall, A. G.; Grosshans, P. B. Anal. Chem. 1991, 63, $215 \mathrm{~A}-229 \mathrm{~A}$

4. Ijames, C. F.; Wilkins, C. L. J. Ant. Chem. Soc. 1988, 110, $2687-2688$

5. Hunt, D. F.; Shabanowitz, J.: Yates, J. R. III; Zhu, N. Z.; Russell, D. H.; Castro, M. E. Natl. Acad. Sci. USA 1987, 84, $620-623$.

6. Sobuki, T.; Russell, D. H. Proc. Nati. Acad. Sci. USA 1992, 89, $5701-5704$

7. Castoro, J. A.; Koster, C.; Wilkins, C. I. Rapid Comm. Mass Spectrom 1992, 6, 239241.

8. Wilkins, C. L.; Castoro, J. A.; Koster, C. Proceedings of the 40th Annual ASMS Conference on Mass Spectronetry and Allied Topics; Washington, DC, 1992.

9. Henry, K. D.; Quinn, J. P.; McLafferty, F. W. I. Am. Chern. Soc. 1991, 113, 5447-5449.

10. Hanson, C. D.; Castro, M. E.; Russell, D. H.; Hunt, D. F.; Shabanowitz, J. In FTMS: Fonolution, Innovation and Applications; Buchanan, M. V., Fd.; ACS Symposium Series 359; American Chemical Society: Washington, DC, 1989; pp $100-115$.

11. Holliman, C. L.; Rempel, D. L.; Gross, M. L. I. Am. Soc. Mass Spectrom. 1992, 3, 460-463.

12. Rempel, D. L.; Grese, R. P.; Gross, M. L. Int. I. Mass Spectrom. Ion Processes 1990, 100, 381-396.

13. Jeffries, J. B.; Barlow. S. E.; Dunn, G. H. Int. J. Muss Spectrom. Ion Processes 1983, 54, 169187.

14. Wang, T. L.; Marshall, A. G. Int. I. Mass Spectrom. Ion Processes 1986, 68, 287-301.

15. Chen, S.; Comisarow, M. B. Rapid Commun. Mass Spectrom. 1991, 5, 450-455.

16. Chen, S.; Comisarow, M. B. Rapid Commun Mass Spectrom. 1992, 6, 1-3.

17. Wang, M.; Marshall, A. G. Int. J. Mass Spectrom. Ion Processes $1990,100,323-346$.

18. Schumacher, R. Intto to Magnetic Resomance; Benjamin: Now York 1970; pp 64-67.

19. Black, H. S. Modulation Theary; Van Nostrand: New York, 1953; $p 195$.

20. Lebrilla, C. B.; Amster, I. J.; McIver, R. T. Int. J. Mass Spectrom. Ion Processes 1989, 87, R7 R13.

21. Hogan, J. D.; Laude, D. A. Jr. Anal. Chem. 1990, 62, 530-535. 